\title{
3D Printing-Assisted Skull Base Tumor Surgeries: An Institutional Experience
}

\author{
Sanjeev Chopra ${ }^{1}$ Ashim Kumar Boro ${ }^{1}$ Virendra Deo Sinha ${ }^{1}$ \\ ${ }^{1}$ Department of Neurosurgery, Sawai Man Singh Medical College, \\ Jaipur, India

\begin{abstract}
Address for correspondence Virendra Deo Sinha, Department of Neurosurgery, Sawai Man Singh Medical College, Jaipur 302004, India (e-mail: sinhavd@yahoo.com).
\end{abstract}

J Neurosci Rural Pract 2021;12:630-634.

\begin{abstract}
Keywords

- skull base

- 3D printing

- 3D tumor model

Three-dimensional (3D) printing technology in neurosurgery has gained popularity nowadays. Skull base contains many major neurovascular structures in a confined space, along with anatomical variations making surgical approaches to this region challenging. 3D-printed model of skull base tumors consists of the patient's bony skull base, actual tumor dimensions, and surrounding major neurovascular structures. We included a total number of five patients with skull base tumors (one case of planum sphenoidale meningioma, two cases of sellar tumor with suprasellar extension, and two cases of cerebellopontine angle tumor) and 3D-printed tumor model of each of them. These models were used for preoperative simulation and served as very true to life training tool. These help in increasing the efficacy of the surgeon, improves surgical safety and ergonomics. They were also used for patient counselling, educating about the disease, the surgical procedure, and associated risks.
\end{abstract}

\section{Introduction}

Application of three-dimensional (3D) printing technology in neurosurgery have gained popularity nowadays. Tumors of the skull base are commonly benign compared with those in other anatomical locations and complete excision leads to good outcome. ${ }^{1}$ Skull base contains many major neurovascular structures in a confined space, along with anatomical variations making surgical approaches to this region challenging. ${ }^{2}$ Development of surgical training devices to improve the required surgical skills along with the precise diagnosis and proper surgical plans are needed to reduce surgical complications. ${ }^{3}$ Micro-neurosurgical techniques have undergone rapid advancement with shifting of attention in skull base surgery to preserve important cranial blood vessels and nerves along with maximal tumor resection. ${ }^{4}$ Traditionally surgeon's anatomical knowledge, surgical skills, and experience, aided with intraoperative neurophysiologic monitoring and neuronavigation were utilized for proper tumor resection and protecting surrounding structures. ${ }^{5,6}$ Surgeons have relied on cadaver specimens for better anatomical mileage and honing their surgical skillset, but cadavers are difficult to acquire. This technology allows surgeons to develop their operative precision. Here patients imaging data can be compiled into a 3D-printed model allowing a more practical assessment along with simulation preoperatively. ${ }^{1,4}$ The development of 3D models by rapid prototyping technique is being increasingly utilized in many surgical specialities. ${ }^{7}$ Benefit of this technology in improving surgical blueprint, aiding in diagnostic quality, decreasing blood loss, and shortening operating time have been favorably reported. ${ }^{8}$ Researches on 3D-printed tumor models along with cranial nerves are not being frequently reported. This is the first reported series of 3D-printed skull base tumors (five cases) in our country as per our knowledge. published online September 16, 2021
DOI https://doi.org/ 10.1055/s-0041-1734001. ISSN 0976-3147.

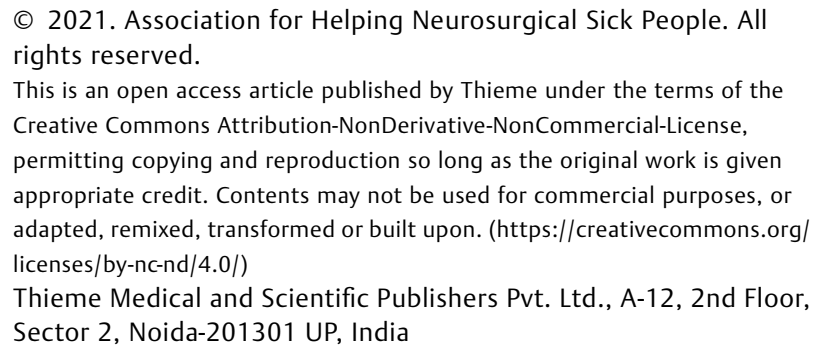

(C) 2021. Association for Helping Neurosurgical Sick People. All rights reserved.

This is an open access article published by Thieme under the terms of the Creative Commons Attribution-NonDerivative-NonCommercial-License, permitting copying and reproduction so long as the original work is given appropriate credit. Contents may not be used for commercial purposes, or adapted, remixed, transformed or built upon. (https://creativecommons.org/ licenses/by-nc-nd/4.0/)

Thieme Medical and Scientific Publishers Pvt. Ltd., A-12, 2nd Floor, Sector 2, Noida-201301 UP, India 


\section{Materials and Methods}

Five patients with skull base tumors (one case of planum sphenoidale meningioma, two cases of sellar tumor with suprasellar extension, and two cases of cerebellopontine angle tumor) with unusual characteristics underwent surgery after preoperative simulation with 3D-printed models at our institute, department of neurosurgery SMS Medical College, Jaipur. The inclusion criteria used were: (1) tumor located at skull base region, (2) tumor abutting nearby neurovascular structures (wrapped, pushed, or infiltrated), (3) complete clinical information, and (4) pathological confirmation of tumor. Variables such as age, gender, size and site of lesion, volume of resection, duration of operation, blood loss, final histopathological diagnosis along with duration of hospitalization were also evaluated. Summary of cases and outcomes are shown below ( - Table $\mathbf{1}$ ).

\section{Data Acquisition Methods}

In every case, patients' imaging data were acquired by various techniques including magnetic resonance imaging (MRI), computed tomography (CT), computed tomography angiography (CTA), and diffusion tensor imaging. All CT, CTA, MRI, and DTI image data were stored in the standard format according to Digital Imaging and Communication in Medicine (DICOM). Then analysis and segmentation of images were done. This was followed by a conversation of DICOM data to standard tessellation language (STL) file format, finally optimization along with cleanup of STL file to obtain high-quality 3D model. The MIMICS 3D image reconstruction software was used to process the image data. First CT scan file was uploaded into MIMICS and segmentation of skull bone was done. The 3D object created can be further modified such as the opening of skull bone can be done and then saved as STL file. Next, CTA images were imported to MIMICS and saved. Similarly, a 3D model of tractography DICOM files was created. Tissues such as bone, tumor, vessels, and nerves were coded with different colors in the model. The resulting 3D model was then saved as STL file. These STL files were sent to $3 \mathrm{D}$ printing machine and the 3D tumor model printed having different colors for different components. Color coding was done both manually and by machine.

\section{Discussion}

Three-dimensional printing technology is an evolving technology for scientific research in the medical field ${ }^{8}$ and has aided in making prognosis better in most delicate and complex surgeries in neurosurgery. Skull base surgery is difficult because of limited operating space with surrounding important neurovascular structures. ${ }^{9}$ In 2013 Oishi et al $^{21}$ reported simulation of operation on a 3D-printed skull base model prototype. 3D printing model of skull base tumors consists of the patient's bony skull base, actual tumor dimensions, and surrounding major neurovascular structures. These models perfectly exhibit the structural relations and help us to pre-plan operative approaches, perform simulated surgery on them, and to scrutinize the potential problems along with the extent of possible

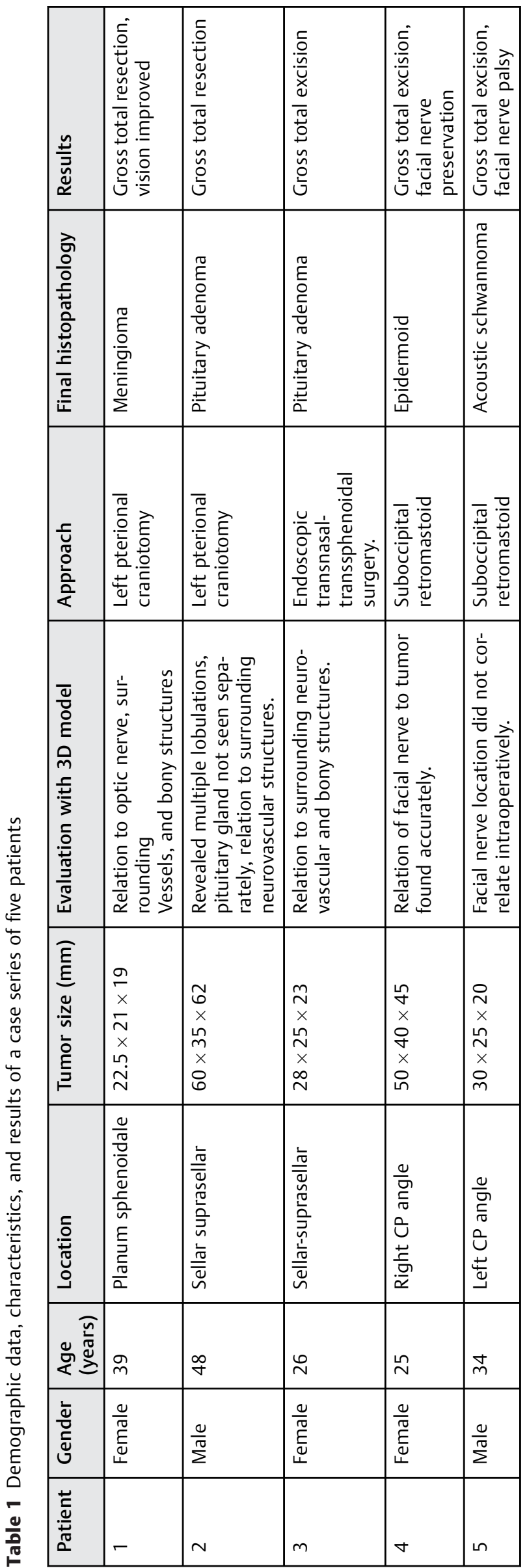




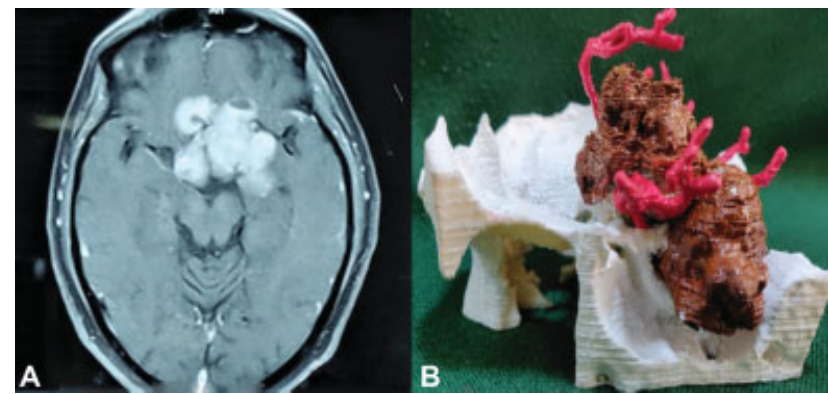

Fig. 1 (A) Axial image of MRI brain postcontrast showing recurrent sellar suprasellar mass and (B) 3D-printed model of the recurrent sellar-suprasellar mass (brown) with blood vessels (red) and bony (white) relations. 3D, threedimensional; MRI, magnetic resonance imaging.

resection protecting surrounding neurovasculatures. Our 3D models were made of nontransparent materials which were flawed in certain aspects. Transparent material to 3D print tumors can be helpful to see the neurovascular structures both inside and outside the tumor. This article describes the use of 3D-printed solid models to recreate complex sellar region, cerebellopontine (CP) angle tumors, and a planum sphenoidal region tumor along with cranial nerves and major vessels. For tumors in the sellar-suprasellar region, relationship of ICA (internal cerebral artery) and optic chiasma with the tumor is important. ${ }^{10}$ The spatial position of the blood vessels and cranial nerves-pushed, wrapped or infiltrated by the tumors can be 3D printed and the best surgical route for excision can be decided. Appropriate parameters like the distance of anterior inferior chiasma from tuberculum sellae are very important for approaches to the suprasellar region as in the case of lateral approach to the region. ${ }^{11}$ This can be very well represented in a life-size 3D-printed model ( $\mathbf{- F i g s . ~} \mathbf{1}$ and $\mathbf{2}$ ). We found it very useful for accessing the thickness of sellar walls, the appearance of sella turcica along with the distance between the anterior sellar wall and tumor mass which helped in a proper presurgical simulation. For the planum sphenoidal tumor, we threedimensionally printed the anterior skull base along with the tumor, surrounding vessels, and optic nerve ( - Fig. 3 ) which was very useful in preoperative simulation.

Approaches like the suboccipital-retrosigmoid approach are among the most common approaches pre-

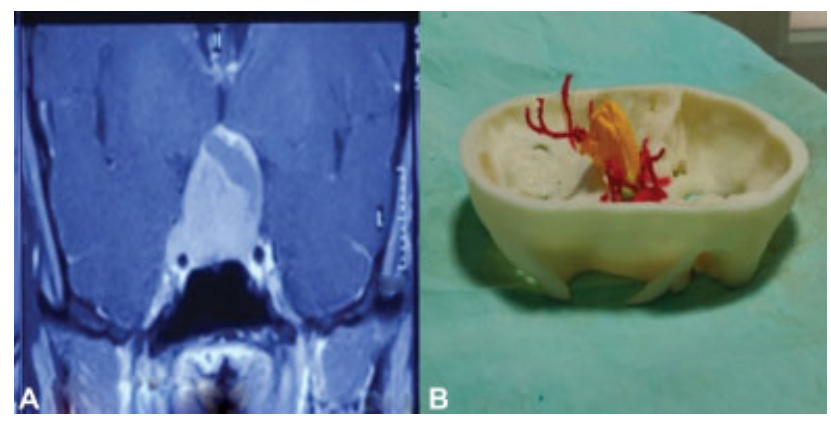

Fig. 2 (A) Coronal images of MRI sella showing sellar-suprasellar mass and (B) 3D-printed model of the sellar-suprasellar mass (yellow) with blood vessels (red), and bony (white) relations. 3D, three-dimensional; MRI, magnetic resonance imaging.

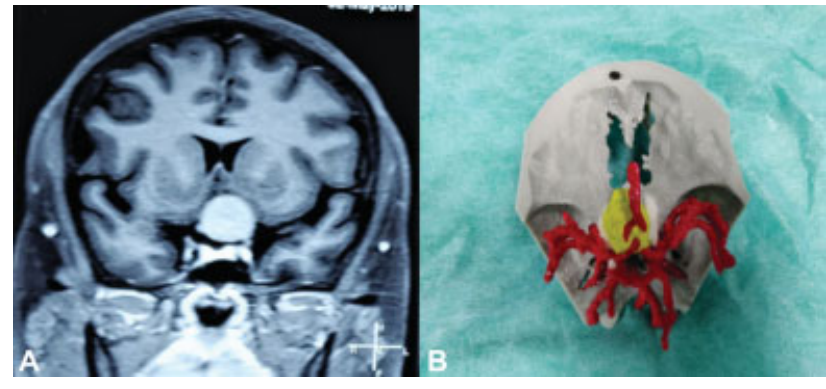

Fig. 3 (A) MRI brain with contrast (coronal image) showing planum sphenoidale meningioma and (B) 3D-printed model of the planum sphenoidal meningioma (yellow) with surrounding major blood vessels (red) and optic nerve (white). 3D, three-dimensional; MRI, magnetic resonance imaging.

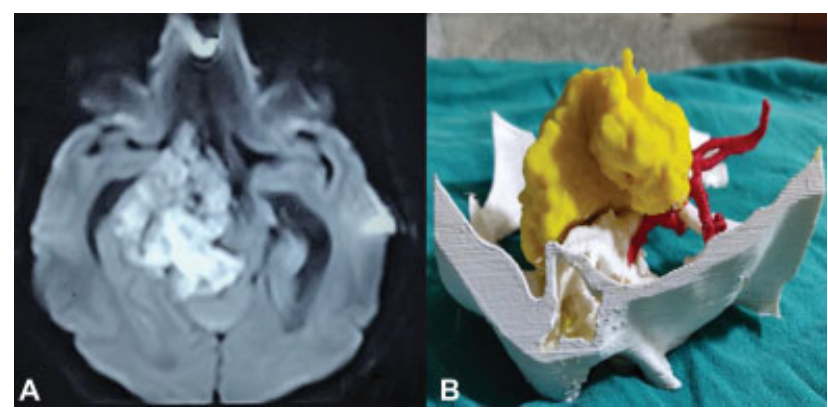

Fig. 4 (A) MRI DW image of brain showing right CP angle epidermoid and (B) 3D-printed model of right CP angle epidermoid (yellow) with its blood vessel (red), and bony (white) relations. 3D, three-dimensional; CP, cerebellopontine; DW, diffusion weighted; MRI, magnetic resonance imaging.

ferred for CP angle tumors. ${ }^{12}$ In this region several important cranial nerves and blood vessels lie nearby providing a narrow operating space. 3D-printed CP angle tumor models can determine the location of bony landmarks and the size of the cranial opening needed to open the operating space via simulation (-Figs. 4 and $\mathbf{5}$ ). Most of the tumors in this region are acoustic schwannoma and the goal of surgery is adequacy of tumor removal along with tumor removal in internal acoustic meatus with facial nerve preservation. ${ }^{13}$ We used DTI for

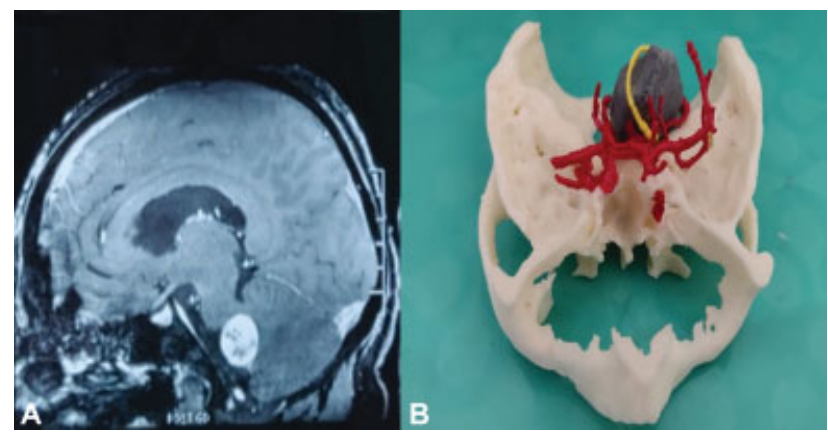

Fig. 5 (A) MRI brain with contrast enhancement (sagittal image) showing left $\mathrm{CP}$ angle schwannoma and (B) 3D-printed model of left $\mathrm{CP}$ angle mass (gray) with cranial nerve (yellow) and blood vessel (red), and bony (white) relations. 3D, three-dimensional; $\mathrm{CP}$, cerebellopontine; MRI, magnetic resonance imaging. 
reconstruction of seventh cranial nerve along with 3Dprinted tumor model; it was very useful for preoperative simulation. However, it was not accurate in our case (-Fig. 5). Our model also could not predict the degree of adhesion of tumor with the neurovascular structures. Hardness of the tumor could not be represented for preoperative simulation. In our 3D models we found delineation of the vessels was better than nerves. Restoring the skull base after surgery is a major hindrance. Incomplete reconstruction may lead to cerebrospinal fluid leakage (rhinorrhea, otorrhea), pneumocephalus, or meningitis. Improper reconstruction thus affects the recovery of the patient postoperatively. ${ }^{14}$ Various methods used for skull base tumor include pedicled fascial flap or titanium plates. ${ }^{15}$ Previously this reconstruction of the base along with duramater was used to be planned intraoperatively but nowadays proper presurgical planning can be executed with 3D-printed models. New researches have been carried out to develop small fabricated units as cell aggregate or biochemical to produce structures such as adipose tissue, muscle, bone, or cartilages by using bioprinting technology to prevent CSF leak. ${ }^{16}$ 3D-printed models help the doctors to communicate better with the patient and their relatives regarding the disease. ${ }^{17}$ We used these models to educate and counsel the patient and their relatives about the tumor location, operative plan, and risks during surgery. Young neurosurgeons can use these models as a training tool as well as to device surgical strategies. ${ }^{18}$ The stereo evaluation of 3D data along with preoperative simulation of neurosurgery can give the surgeon additional confidence while working with complex surgical anatomies. ${ }^{19}$

\section{Limitations and Future Prospects}

We noticed that 3D-printed models with cranial nerves were not always comparable with per-operative findings. This model is useful for determining the position of the fiber bundle and their displacement by the tumor. We used DTI to print nerve fibers. DTI, based on the diffusion of water molecules, ${ }^{20}$ may be altered by tumor compression on the nerve fibers affecting density and integrity. This could affect accurate representation and printing of the cranial nerve extracted from DTI data. Intraoperative drainage of cerebrospinal fluid leads to relaxation of brain changes the position of the tumor along with surrounding neurovascular structures in comparison to simulated 3D-printed tumor model. This dynamic process could not be recreated and incorporated in our models which were made of rigid materials, so future designs will need to address these problems. Moreover, MRI studies presently can show only major vascular structures and smaller ones $(<1 \mathrm{~mm}$ in diameter) are not captured, so could not be printed. To avoid complications, intraoperatively bleeding monitoring from these vessels need to be done. Our study consisted of five cases only which makes a very small series. For a more thorough evaluation of this technique, greater number of randomized controlled trials with multiple centers involvement needs to be done for better future prospects and acceptance.

\section{Conclusion}

Our 3D-printed skull base tumor models were made with DICOM data compiled from CT, CTA, MRI, and DTI studies. The final 3D-printed models had positional relationships of cranial nerves and blood vessels to the tumor mass along with useful bony landmarks. These models facilitated preoperative simulation and served as very true to life training tool, assisting the surgeon in positioning and allowing better identification of severely displaced structures like cranial nerves and blood vessels. Thus these 3D-printed models increase the efficacy of the surgeon, improves surgical safety and ergonomics. They can also be used for patient counselling, educating about the disease, the surgical procedure, and associated risks.

\section{Conflict of Interest}

None declared.

\section{References}

1 Maroon JC. Skull base surgery: past, present, and future trends. Neurosurg Focus 2005;19(01):E1

2 Solari D, Villa A, De Angelis M, Esposito F, Cavallo LM, Cappabianca P. Anatomy and surgery of the endoscopic endonasal approach to the skull base. Transl Med UniSa 2012;2:36-46

3 Sekhar LN, Juric-Sekhar G, Qazi Z, et al. The future of skull base surgery: a view through tinted glasses. World Neurosurg 2020; 142:29-42

4 Lin J, Zhou Z, Guan J, et al. Using three-dimensional printing to create individualized cranial nerve models for skull base tumor surgery. World Neurosurg 2018;120:e142-e152

5 Kim K, Cho C, Bang MS, Shin HI, Phi JH, Kim SK. Intraoperative neurophysiological monitoring: a review of techniques used for brain tumor surgery in children. J Korean Neurosurg Soc 2018;61 (03):363-375

6 Orringer DA, Golby A, Jolesz F. Neuronavigation in the surgical management of brain tumors: current and future trends. Expert Rev Med Devices 2012;9(05):491-500

7 Liaw CY, Guvendiren M. Current and emerging applications of 3D printing in medicine. Biofabrication 2017;9(02):024102

8 Carlstrom LP, Perry A, Graffeo CS, Driscoll CL, Link MJ, Morris JM. A model of success: incorporating 3D-printed models of skull base pathology for surgical approach planning. J Neurol Surg B Skull Base 2020;81(01):252

9 Guo XY, He ZQ, Duan H, et al. The utility of 3-dimensional-printed models for skull base meningioma surgery. Ann Transl Med 2020; 8(06):370

10 Ottenhausen M, Banu MA, Placantonakis DG, et al. Endoscopic endonasal resection of suprasellar meningiomas: the importance of case selection and experience in determining extent of resection, visual improvement, and complications. World Neurosurg 2014;82(3-4):442-449

11 Agazzi S, Youssef AS, van Loveren HR. The anterolateral approach for the transcranial resection of pituitary adenomas: technical note. Skull Base 2010;20(03):143-148

12 Nayak P, Kumar R. Retromastoid-sub occipital: a novel approach to cerebello pontine angle in acoustic neuroma surgery-our experience in 21 cases. J Neurosci Rural Pract 2011;2(01): $23-26$ 
13 Memari F, Hassannia F, Abtahi SH. Surgical outcomes of cerebellopontine angle tumors in 50 cases. Iran J Otorhinolaryngol 2015; 27(78):29-34

14 Rodrigues M, O'malley BW Jr, Staecker H, Tamargo R. Extended pericranial flap and bone graft reconstruction in anterior skull base surgery. Otolaryngol Head Neck Surg 2004;131(01): 69-76

15 Khan A, Lapin A, Eisenman DJ. Use of titanium mesh for middle cranial fossa skull base reconstruction. J Neurol Surg B Skull Base 2014;75(02):104-109

16 Kolesky DB, Truby RL, Gladman AS, Busbee TA, Homan KA, Lewis JA. 3D bioprinting of vascularized, heterogeneous cell-laden tissue constructs. Adv Mater 2014;26(19):3124-3130

17 Andolfi C, Plana A, Kania P, Banerjee PP, Small S. Usefulness of three-dimensional modeling in surgical planning, resident train- ing, and patient education. J Laparoendosc Adv Surg Tech A 2017; 27(05):512-515

18 Yang T, Tan T, Yang J, et al. The impact of using three-dimensional printed liver models for patient education. J Int Med Res 2018;46 (04):1570-1578

19 Ploch CC, Mansi CSSA, Jayamohan J, Kuhl E. Using 3D printing to create personalized brain models for neurosurgical training and preoperative planning. World Neurosurg 2016;90:668-674

20 Price SJ, Burnet NG, Donovan T, et al. Diffusion tensor imaging of brain tumours at 3T: a potential tool for assessing white matter tract invasion? Clin Radiol 2003;58(06):455-462

21 Oishi M, Fukuda M, Yajima N, Yoshida K, Takahashi M, Hiraishi T, Takao T, Saito A, Fujii Y. Interactive presurgical simulation applying advanced 3D imaging and modeling techniques for skull base and deep tumors. Journal of neurosurgery 2013;119(01):94-105 